\title{
CXCL2-mediated ATR/CHK1 signaling pathway and platinum resistance in epithelial ovarian cancer
}

\author{
Sipei Nie ${ }^{\dagger}$, Yicong Wan ${ }^{\dagger}$, Hui Wang ${ }^{\dagger}$, Jinhui Liu ${ }^{\dagger}$, Jing Yang, Rui Sun, Huangyang Meng, Xiaolin Ma, Yi Jiang and \\ Wenjun Cheng ${ }^{*}$ (D)
}

\begin{abstract}
Tumor microenvironment and chemokines play a significant role in cancer chemoresistance. This study was designed to reveal the important role of CXCL2 in platinum resistance in epithelial ovarian cancer (EOC). Differently expressed (DE) genes were screen out based on analysis of GSE114206 dataset in GEO database. The expression of DE chemokines was further validated in platinum- resistant and sensitive EOC. Cell viability assay and cell apoptosis assay were performed to explore the roles of CXCL2 in EOC. Cell stemness characteristics and the signaling pathway regulated by CXCL2 were also investigated in this study. As the results showed, CXCL2 was identified up-regulated in platinum-resistant EOC. The functional assays showed overexpressing CXCL2 or cO-culturing with recombinant human CXCL2 promoted cell resistance to cisplatin. Conversely, knocking down CXCL2 or co-culturing with neutralizing antibody to CXCL2 increased cell response to cisplatin. CXCL2 overexpressing maintained cell stemness and activated ATR/CHK1 signaling pathway in EOC. Moreover, we further demonstrated that CXCL2-mediated resistance to cisplatin could be saved by SB225002, the inhibitor of CXCL2 receptor, as well as be rescued by SAR-020106, the inhibitor of ATR/CHK1 signaling pathway. This study identified a CXCL2-mediated mechanism in EOC platinum resistance. Our findings provided a novel target for chemoresistance prevention in EOC.
\end{abstract}

Keywords: Epithelial ovarian cancer (EOC), Platinum-resistance, Chemokine, CXCL2

\section{Backgrounds}

Epithelial ovarian cancer (EOC) is the first cause of gynecological malignancy-related death [1]. At present, platinum-based chemotherapies were recommended as the first-line chemotherapeutic regimens for EOC. Although the tremendous progress has made in comprehensive therapies, the survival rate of EOC patients with advanced tumor remains poor even in highresource countries such as the United States and Canada [2]. Though initial response rates of platinum-based

\footnotetext{
*Correspondence: wenjunchengdoc@163.com

${ }^{\dagger}$ Sipei Nie, Yicong Wan, Hui Wang and Jinhui Liu contributed equally to this work.

Department of Gynecology, the First Affiliated Hospital of Nanjing Medical University, Nanjing 210029, Jiangsu, China
}

regimens are $60-80 \%$, majority of EOC patients acquire platinum resistance during treatment. Platinum resistance is one of the primary causes of subsequent relapses and metastasis [3]. Thus, platinum resistance remains an urgent challenge for EOC patients, but the mechanism remains unidentified. There is a lack of effective approach to against platinum resistance in malignancies. Therefore, revealing the molecular mechanism contributing to platinum resistance in EOC and exploring therapeutic targets are clinical significances.

Chemokines contain a group of about 50 small (8$14 \mathrm{kDa})$ secreted proteins. These molecules regulate cell biological processes, including in malignancies initiation and progression $[4,5]$. These secreted proteins work by interacting with the corresponding receptors, original author(s) and the source, provide a link to the Creative Commons licence, and indicate if changes were made. The images or other third party material in this article are included in the article's Creative Commons licence, unless indicated otherwise in a credit line to the material. If material is not included in the article's Creative Commons licence and your intended use is not permitted by statutory regulation or exceeds the permitted use, you will need to obtain permission directly from the copyright holder. To view a copy of this licence, visit http://creativecommons.org/licenses/by/4.0/. The Creative Commons Public Domain Dedication waiver (http://creativeco mmons.org/publicdomain/zero/1.0/) applies to the data made available in this article, unless otherwise stated in a credit line to the data. 
a family of about 20 seven-transmembrane G-proteincoupled receptors [6]. Previous studies have suggested chemokines and their corresponding receptors were involved in malignancies progression mainly in the three mechanisms: attracting cancer cells for metastasis; mobilization of hematopoietic cell populations from the bone marrow to colonize at the tumor site and regulate tumor processes; acting as growth factors and supporting tumor growth through an autocrine pathway [6, 7]. Increasing evidences have supported that using immune checkpoint inhibitors that targeting chemokines and their receptors becomes a novel approach of cancer therapy [8-10]. The anti-CCR4 monoclonal antibody and the CXCR4 receptor inhibitor are already in the clinical practice for hematological malignancies [11-15]. CXCL10 was suggested as an immune checkpoint molecule in cancer, displaying a positive autocrine effect and directly suppressing tumor growth[16]. CXCL17 expression was that associated with lung cancer and hepatic cancer[17]. In addition, recently studies also proved chemokines and their receptors participate in the cancer chemoresistance. Ren et al. demonstrated CXCR3-mediated AMPK signaling pathway contributed to metabolic alteration during the chemoresistance to agent-sorafenib in hepatocellular carcinoma [18]. Zhang et al. identified CXCL13 was involved in 5-Fu resistance in colorectal cancer [19]. These studies suggested that chemokines and their receptors might be potential novel therapeutic targets of cancer chemoresistance.

Thus, the present study devoted to exploring the role of chemokines in platinum-resistant EOC and investigate the biological function and underlying regulation mechanism of the candidate chemokine. Currently, gene expression profiles have been increasingly used to identify candidate significant genes in various diseases, especially in malignancies [20]. Public genomics data repositories provided powerful systems biology approaches to detect the association between genes and cancer. In the present study, we devoted to exploring the role of chemokines in platinum-resistant EOC. We used bioinformatics methods to screen our differentially expressed (DE) chemokines by comparing gene expression profiles based on platinum sensitivity status and ADP-ribose polymerase (PARP) levels. Candidate DE chemokines were next validated in platinum-resistant and sensitive EOC samples and cells.

\section{Materials and Methods}

\section{Differentially expressed genes (DEGs) screening in EOC with platinum resistance}

The gene expression profile was obtained from GSE114206 dataset of GEO database. GSE114206 dataset contained 6 low PARP and platinum-resistant EOC samples and 6 high PARP and platinum-sensitive EOC samples. We use the "limma" $\mathrm{R}$ package to screen the DEGs between the platinum- resistant and sensitive EOC samples. Adjusted $P$-value $<0.05$ and $\mid \log 2$ fold change (FC) $\mid>1$, were chosen as the cut-off threshold. Candidate DE chemokines were visualized by "circos" package (https://www.omicstudio.cn/tool).

\section{Patients and clinical sample collection}

All patients signed informed consent before using clinical specimens, and the use of specimens for this study has been proved by the ethics committee of the First Affiliated Hospital with Nanjing Medical University. All the ovarian cancer tissues and serum samples were obtained from patients diagnosed and underwent surgery in the First Affiliated Hospital with Nanjing Medical University between January 2015 to January 2020. Surgical specimens were immediately at $-80{ }^{\circ} \mathrm{C}$ for nucleic acid and protein extraction. Patient who did not achieve the clinical complete response in the initial therapy or whom with recurrent tumor within 6 months was identified as an primary resistance case [21]. Due to the secondary operation is not recommended in patients with recurrent resistant tumor, collected samples in our study were limited (15 platinum-sensitive and 9 platinum-resistant EOC samples). Meanwhile, serum samples of 6 platinumresistant and 9 platinum-sensitive EOC patients were also collected.

\section{Cell culture and chemicals}

HO8910 and A2780 are platinum-sensitive EOC cells. By exposure to increasing concentration of cisplatin, the cisplatin-resistant cells A2780-DDP, HO8910-DDP were constructed in our study for further research. FBS (CLRAK Bioscience) and 1\% penicillin/streptomycin (Sigma-Aldrich) at $37^{\circ} \mathrm{C}$ supplied with $5 \% \mathrm{CO} 2$.

\section{Plasmid and siRNA transfection}

The plasmid encoding the transcript of CXCL2 (oeCXCL2) and the negative control (oeNC) was synthesized by Tsingke (Nanjing, China). The siRNA targeted CXCL2 (siCXCL2) and the negative control (siNC) were purchased from GemmaPharma (Shanghai, China). Cells were transfected by Lipofectamine 3000 Transfection Reagent (L3000150, Invitrogen) for 24-48 h. The CXCL2 siRNA sequence was described in the previous study [22].

\section{Cell viability assay}

About 6000-8000 preprocessed EOC cells were plated in 96-well plates, and incubated in a series of cisplatin concentration $(0,5,10,15$ and $20 \mu \mathrm{M})$ (Sigma-Aldrich) for 48 h. Cell viability was qualified by Cell Counting Kit- 8 
(CCK8) (Vazyme) under the manufacturer's instructions. A microplate reader (TECAN, Infinite M200 PRO) was applied to measure the absorbance at $450 \mathrm{~nm}$, and the cell' $\mathrm{IC}_{50}$ were analyzed in Graphpad 8.0.

\section{RNA extraction and quantitative Real-time PCR (qRT-PCR)}

According to the manufacturer's instructions, the total RNA of cultured cells and EOC tissues was extracted with Trizol (Invitrogen). cDNA HiScript Q RT SuperMix for qRT-PCR (Vazyme) were used to prepare cDNA. qRT-PCR was performed under the instructions of SYBR Green PCR Kit (Vazyme). The sequences of gene primers used for qRT-PCR were synthesized by Tsingke (Nanjing, China) (Supplementary Table 1). The mRNA expression level was normalized to GAPDH to calculate the relative gene expression.

\section{Western blot assay}

Firstly, cultured cells were lysed in RIPA buffer (Beyotime) with protease inhibitor (Beyotime). Proteins were then extracted and quantified. Western blot assay was performed by the protocol that we previously reported [23]. The reagents and antibodies were shown in Supplementary Table 2 .

\section{Enzyme-linked immunosorbent assay (ELISA)}

Serum samples of platinum-resistant and platinum-sensitive patients and cell culture supernatants were respectively extracted for cytokine analysis. Human CXCL2 ELISA kits were purchased from Hengyuan Biological Co. Ltd (Shanghai, China), and ELISA assay was performed as the manufacturers' instructions. Each sample was duplicated.

\section{Cell apoptosis assay}

EOC cells were plated in 6-well plates and treated with cisplatin $(5 \mu \mathrm{M} / 10 \mu \mathrm{M})$ for $48 \mathrm{~h}$. Then, $2 \times 10^{4}$ cells and the cultural supernatant were collected for apoptosis assay. FITC Annexin V $(5 \mu \mathrm{l})$ and propidium iodide $(5 \mu \mathrm{l})$ (BD Biopharmingen) were used to stain the collected cells by suspending in $300 \mu \mathrm{l}$ of binding buffer for $15 \mathrm{~min}$ in the dark. Cell apoptosis was eventually detected by a flow cytometer (FACScan; BD Biosciences) with Cell Quest software (BD Biosciences).

\section{Statistical analysis}

Each sample was analyzed based on repeated results at least three times and analyzed in Graphpad 8.0. A standard Student's t-test determined the statistical significance of differences between the two groups. Differences at $p<0.05$ were regarded as statistically significant $(*)$, ones at $p<0.01\left(^{* * *}\right), p<0.001\left(^{* * * *}\right)$ or $\left.p<0.00011^{* * * * *)}\right)$ was considered higher statistical significances.

\section{Results}

CXCL2 is up-regulated in platinum-resistant EOC

By analyzing the gene expression profiles of GSE114206 dataset, we identified 1269 up-regulated DEGs and 1140 down-regulated DEGs $(P<0.05, \mid \log 2 \mathrm{FC}>1)$. Among these DEGs, DE chemokines including CXCL2, CXCL11 and CXCL13 were screened out (Fig. 1A). By further validating mRNA expression level of CXCL2, CXCL11 and CXCL13, we found CXCL2 was up-regulated in platinum-resistant EOC samples (Fig. 1B) and cisplatinresistant EOC cells (Fig. 1C-F). Additionally, CXCL2 was also found up-regulated in serum of platinum-resistant EOC patients (Fig. 1G).

CXCL2 overexpression promotes cell resistance to cisplatin After transfecting with plasmids, A2780 and HO8910 with CXCL2 overexpressing and the negative control were constructed (Fig. 1H). Then, cell viability assays showed that CXCL2 overexpression remarkably promoted cancer cell chemoresistance in cisplatin-sensitive EOC cells (Fig. 1I). Oppositely, the cell viability assay illustrated a decreased $\mathrm{IC}_{50}$ in cisplatin-resistant EOC cells with CXCL2 knocking down (Fig. 1J).

\section{CXCL2 promotes cell resistance to cisplatin in EOC by an autocrine mechanism}

We firstly used culture supernatant of A2780-DDP and HO8910-DDP cells to incubate A2780 and HO8910 respectively. The result showed co-cultured cells behaved higher resistance to cisplatin (Fig. 2A and B). Meanwhile, we found CXCL2 was up-regulated in culture supernatant of cisplatin-resistant EOC cells (Fig. 2C). CXCL2 in culture supernatant was also consistently regulated by overexpressing or knocking down CXCL2, which suggested CXCL2 in the tumor microenvironment (TME) might be regulated by autocrine mechanism (Fig. 2D).

\footnotetext{
(See figure on next page.)

Fig. 1 CXCL2 overexpression promotes cell resistance to cisplatin in EOC. A. CXCL2, CXCL11 and CXCL13 were identified as DEGs in GSE114206. B The mRNA expression in platinum-resistant $(n=9)$ compared to platinum-sensitive patients $(n=15)$. C-E The mRNA expression in cisplatin-sensitive and resistant EOC cell lines. F The CXCL2 protein level in cisplatin-sensitive and resistant EOC cell lines. G CXCL2 levels in serum detected by ELISA assay. $\mathbf{H}$ Overexpressing and knocking down efficiencies of transfection. I The cell viability assay showed overexpressing CXCL2 significantly increased IC $\mathrm{C}_{50}$ in EOC cells. J The cell viability assay showed knocking down CXCL2 significantly decreased IC ${ }_{50}$ in cisplatin-resistant EOC cells. Abbreviation: DEG: differentially expressed genes, R: resistant samples; S: sensitive samples
} 


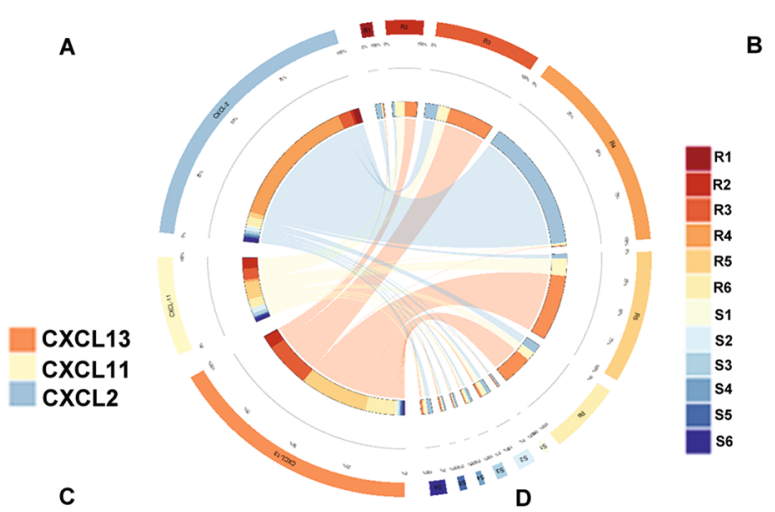

B
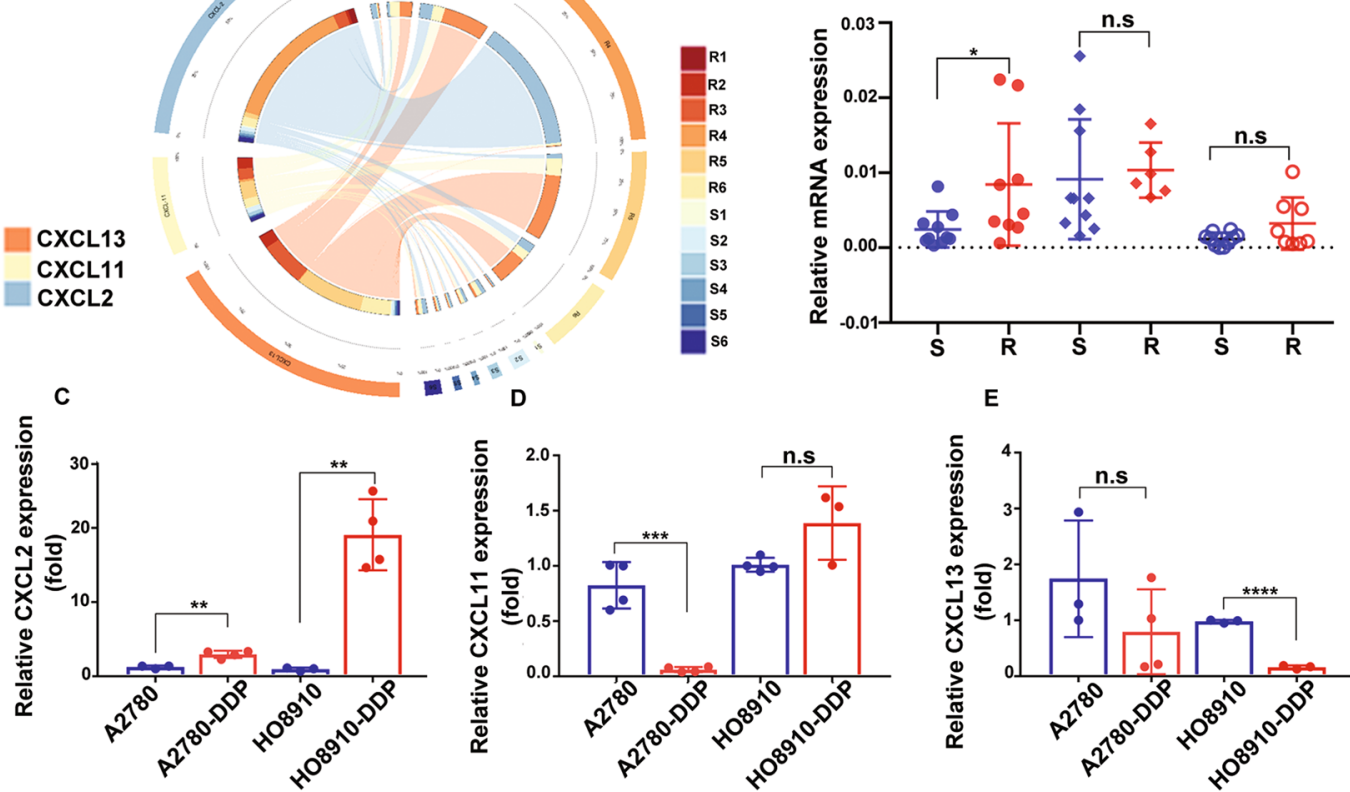

$\mathbf{F}$

G
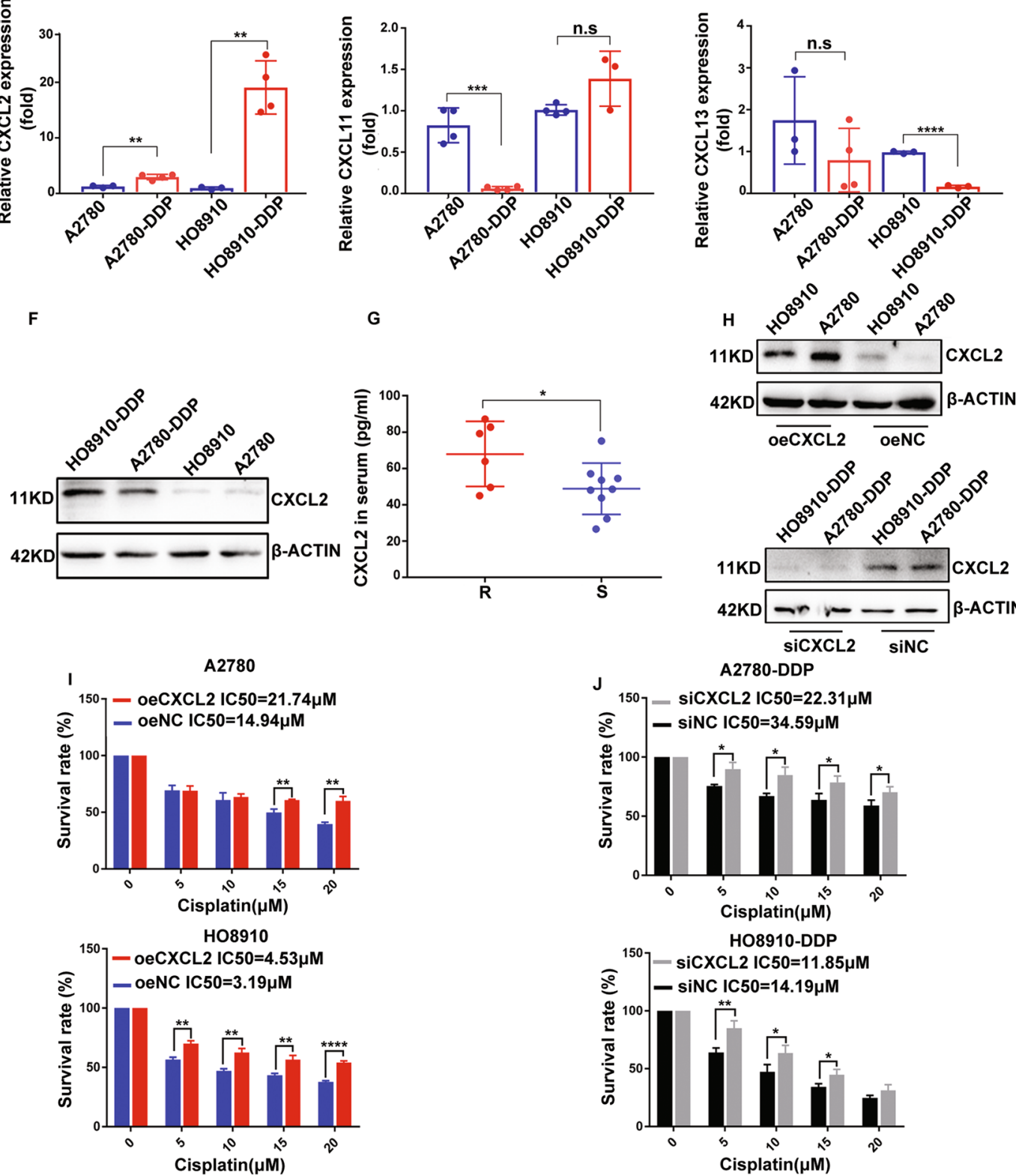

Fig. 1 (See legend on previous page.) 


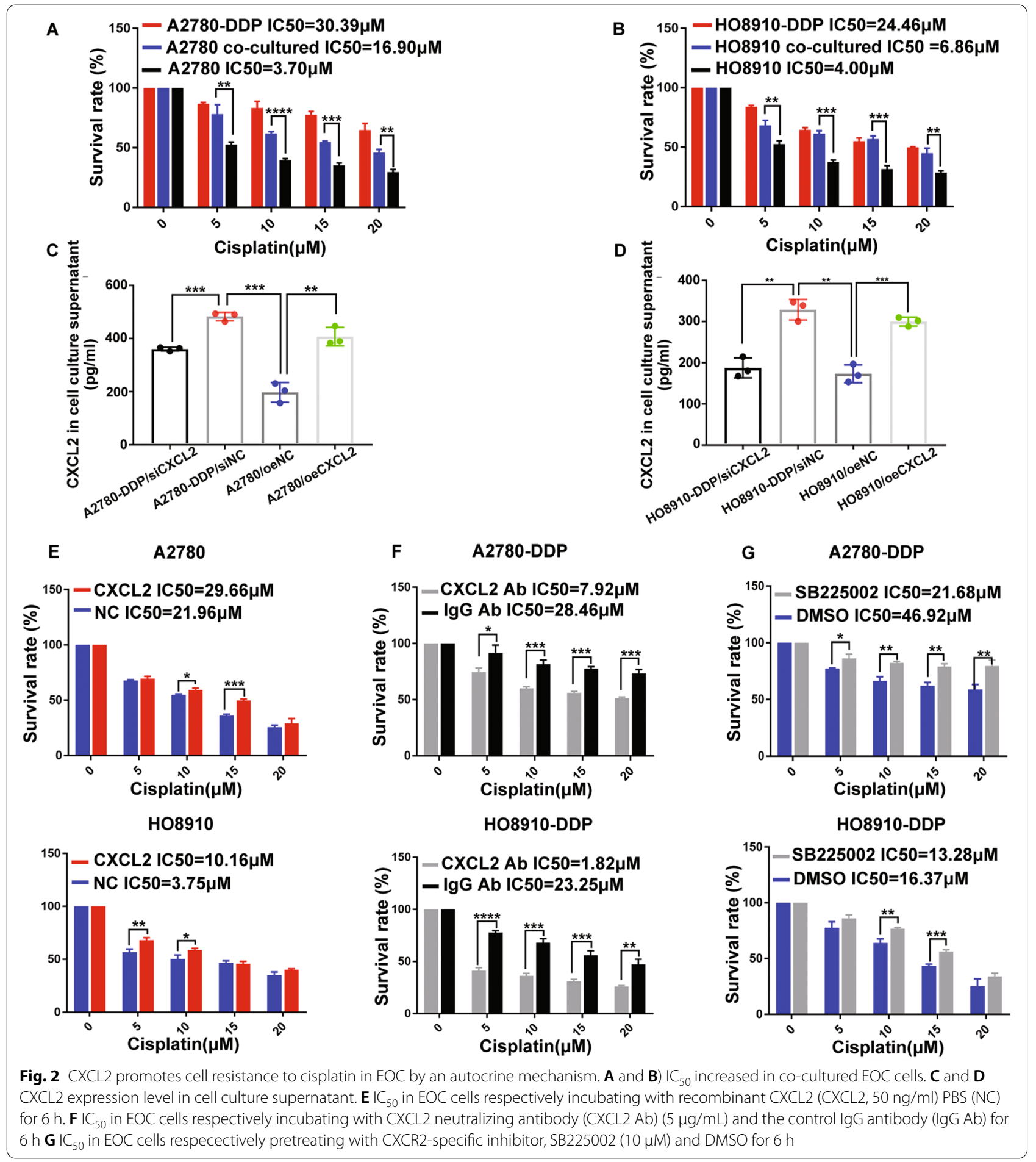

Moreover, the addition of recombinant CXCL2 promoted cell resistance to cisplatin in A2780 and HO8910 (Fig. 2E).Oppositely, incubating with CXCL2 neutralizing antibody weakened the chemoresistance in A2780-DDP and HO8910-DDP (Fig. 2F). These findings showed that
CXCL2 up-regulation in the cell culture environment promoted cell resistance to cisplatin. In addition, we further investigate whether blocking CXCR2, the receptor of CXCL2 could inhibiting cisplatin resistance. By using SB225220, the inhibitor of CXCR2, to incubate 


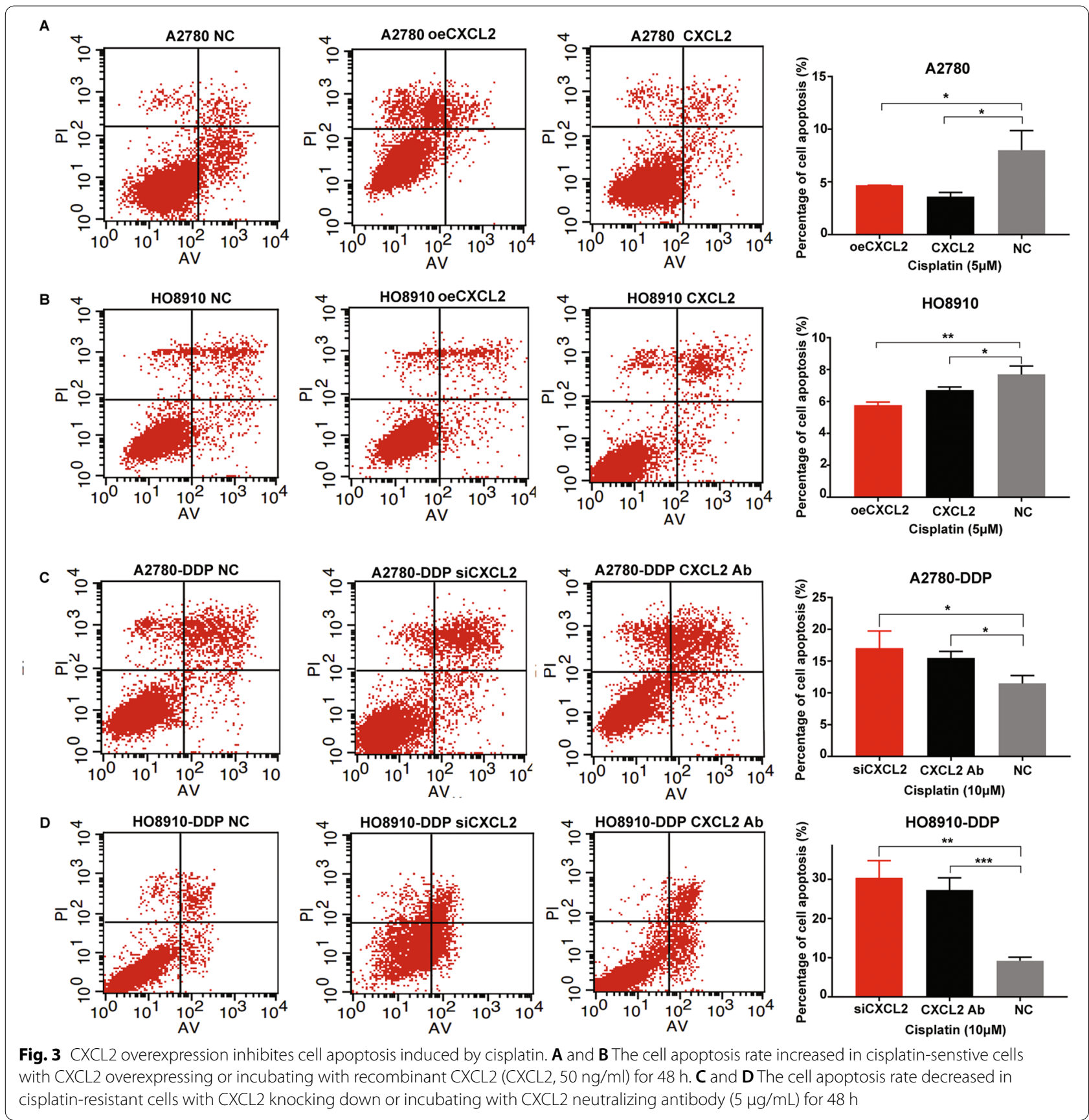

A2780-DDP and HO8910-DDP, we found the $\mathrm{IC}_{50}$ in cisplatin-resistant EOC cells was also decreased (Fig. 2G).

\section{CXCL2 inhibits cell apoptosis and maintains cell stemness in $\mathrm{EOC}$}

The results of cell apoptosis assay showed that the cell apoptosis rate was decreased in cells with CXCL2 overexpressing or cells incubating with recombinant CXCL2
(Fig. 3A and B). On the contrary, cells with CXCL2 knocking down or cells incubating with CXCL2 neutralizing antibody facilitated cell apoptosis after treating with cisplatin (Fig. 3C and D). Meanwhile, we found the cell stemness characteristic, including Nanog, SOX2 and OCT4, was also increased in cells with CXCL2 upregulation, while decreased in cells with CXCL2 downregulation (Fig. 4). 


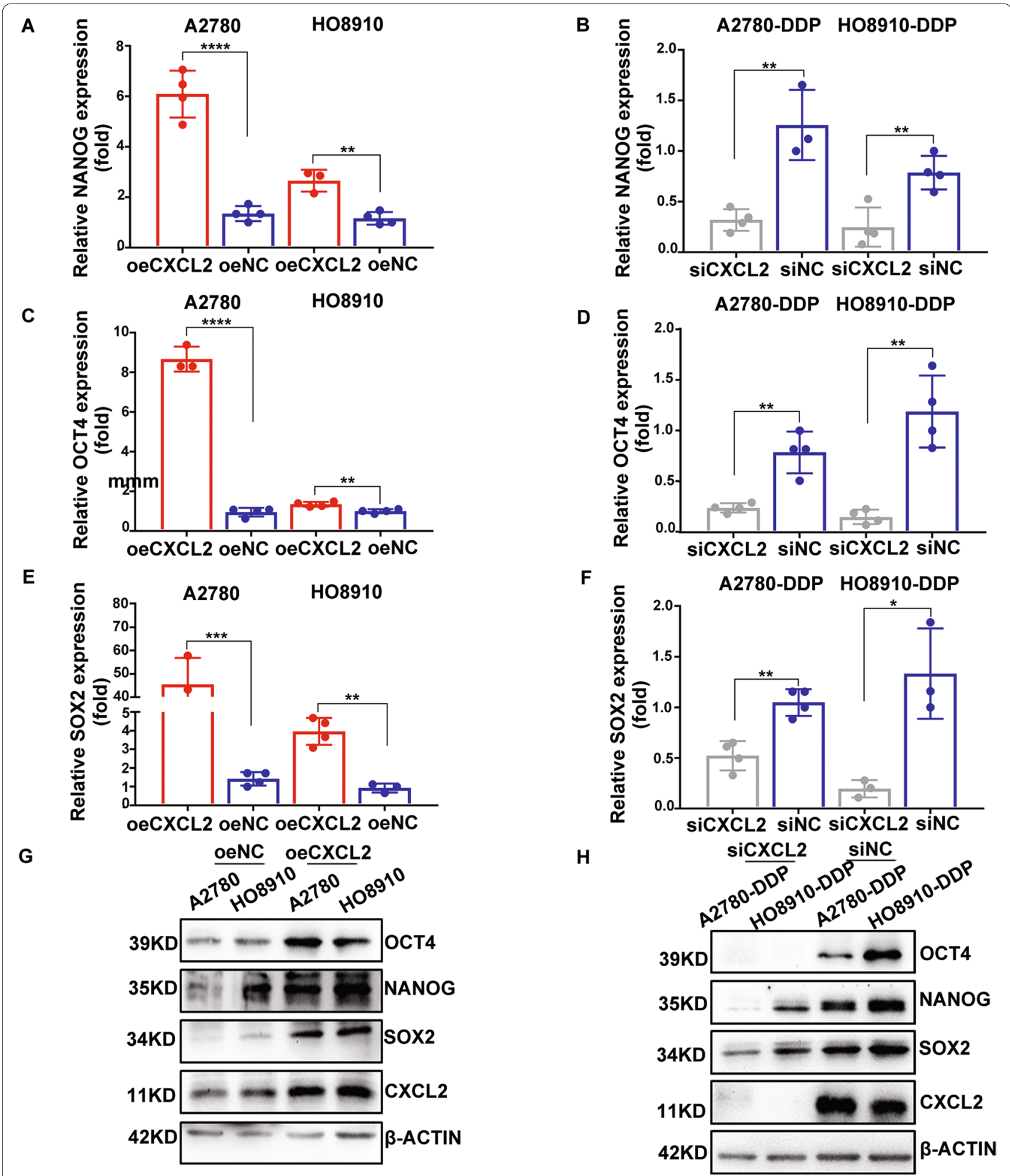

Fig. 4 CXCL2 overexpression maintains cell stemness in EOC. A and B. NANOG mRNA level was positively regulated by CXCL2. C and D OCT4 mRNA level was positively regulated by CXCL2. $\mathbf{E}$ and $\mathbf{F}$ SOX2 mRNA level was positively regulated by CXCL2. $\mathbf{G}$ and $\mathbf{H}$ The results of Western-blot assay showed CXCL2 maintained cell stem characteristics 


\section{CXCL2 promotes cell resistance to cisplatin in EOC by mediating ATR/CHK1 signaling pathway}

Given that the ATR/CHK1 signaling pathway has been proved to play a significant role in DNA damage repairment (DDR) and resulte in cancer chemoresistance. We speculated whether CXCL2 meidated cell resistance to cispaltiin in the ATR/CHK1 signaling pathway. The results showed that ATR and the downstream molecule CHK1 were up-regulated in platinum-resistant EOC samples and cells (Fig. 5A-D). To investigate the effect of ATR/CHK1 signaling pathway activation on EOC platinum resistance, we used SAR-020106, the inhibitor of CHK1, to incubate platinum-resistant cells. The results showed the addition of SAR-020106 weakened the chemoresistance in cisplatin-resistant EOC cells (Fig. 5E and F). Our findings further showed that ATR and CHK1 expressions were consistently up-regulated in cells with CXCL2 overexpressing, while down-regulated in cells with CXCL2 knocking down (Fig. 5G-L). We also analyzed the correlation of CXCL2 and ATR/CHK1 gene expression based on GSE114206 dataset and The Cancer Genome Atlas (TCGA) database. However, the results of correlation analyses showed no statistical significance (Supplementary Fig. 1). Moreover, rescue assays were performed to further demonstrate the effect of CXCL2 mediating-activation of ATR/CHK1 signaling pathway in EOC platinum resistance. The rescue assays displayed the addition of SAR-020106 alleviated the chemoresistance in EOC cells with CXCL2 overexpression (Fig. 5M and $\mathrm{N})$.

\section{Discussions}

TME significantly influences therapeutic response and clinical outcomes of malignancies. According to previous studies, multiple chemokines in TME were demonstrated to be associated with tumor growth, metastasis and cancer cell stemness, which contribute to cancer chemoresistance $[6,24,25]$. In the present study, we devoted to exploring the chemokines involved in EOC resistance to platinum. As a result, we identified candidate chemokines by bioinformatics analysis and identified that the CXCL2 was notably up-regulated in platinum-resistant EOC. CXCL2 overexpression was then demonstrated to promote cancer cell chemoresistance in EOC. The CXCL2 overexpression was further found to inhibit cell apoptosis rate induced by cisplatin and maintain cell stemness in EOC. Mechanistically, we found that CXCL2 promotes EOC cell resistance to cisplatin by mediating ATR/CHK1 signaling pathway.

As the results illustrated, we demonstrated that CXCL2 was up-regulated in platinum-resistant EOC, and itpromotes cell resistance to cisplatin. CXCL2, known as growth-related oncogene- $2 /-\beta$ or macrophage inflammatory protein- $2 \alpha$, is $90 \%$ identical in amino acid sequence [26]. CXCL2 works via the corresponding receptor, CXCR2, and the CXCL2/CXCR2 axis have been extensively studied in malignancies. Previous studies reported CXCL2/CXCR2 axis played a significant role in promoting neutrophils and endothelial cells' chemotaxis, resulting in tumor growth, angiogenesis, chemoresistance, and transformation [27-29]. Makoto et al. suggested omental adipocytes triggered gastric cancer cells proliferation, migration and angiogenesis induction by CXCL2 secretion [30]. Monocytes-derived CXCL2 and CXCL8 are the main causes in regulating neutrophils' recruitment into TME of hepatocellular carcinoma, which could inhibit cancer cell apoptosis [31]. The previous study has also suggested that CXCL2 concentration was up-regulated in the serum of ovarian adenocarcinoma patients with chemoresistance. CXCR2 overexpression has been demonstrated to promote ovarian cancer progression $[32,33]$. However, there is a lack of research about the role of CXCL2 and the regulation mechanism in platinum-resistant EOC. Herein, this study systematically explored the molecule biological function and the underlying mechanism of CXCL2 in platinum-resistant EOC. CXCL2 was proved to protect cell apoptosis from cisplatin treatment and maintain cancer cell stemness, which might result in cell chemoresistance phenotype.

Currently, the formation of DDP-DNA complex, which inhibited DDR is considered as one of the most classical mechanism of cisplatin anti-tumor action [34]. One of the practical approaches to intervene in the DDR process of platinum-resistant cancer is interfering with cell cycle checkpoint signaling pathway [35]. In the present study, CXCL2-mediated ATR/CHK1 signaling pathway was found to promote platinum resistance in EOC. ATR and its downstream kinase CHK1 can be activated by DNA damage and DNA replication stress [36, 37]. Activating ATR/CHK1 signaling pathway results in cell cycle arresting, allowing time for DNA replication and corrected DNA repairment [38]. Besides, activating ATR/ CHK1 signaling pathway stabilizes replication forks and prevents collapse into DNA double-strand breaks [39, 40]. Those functions of ATR/CHK1 signaling pathway provide potential therapeutic targets to overcome cancer therapeutic resistance. ATR/CHK1 inhibitors have been designed and applied singly or paired with radiotherapy and chemotherapy in preclinical and clinical studies, including in EOC [41]. Huntoon et al. suggested that inhibiting ATR/CHK1 broadly sensitizes cancer cells to chemotherapy, which is independent of BRCA status in ovarian cancer [42]. Our findings suggested a 


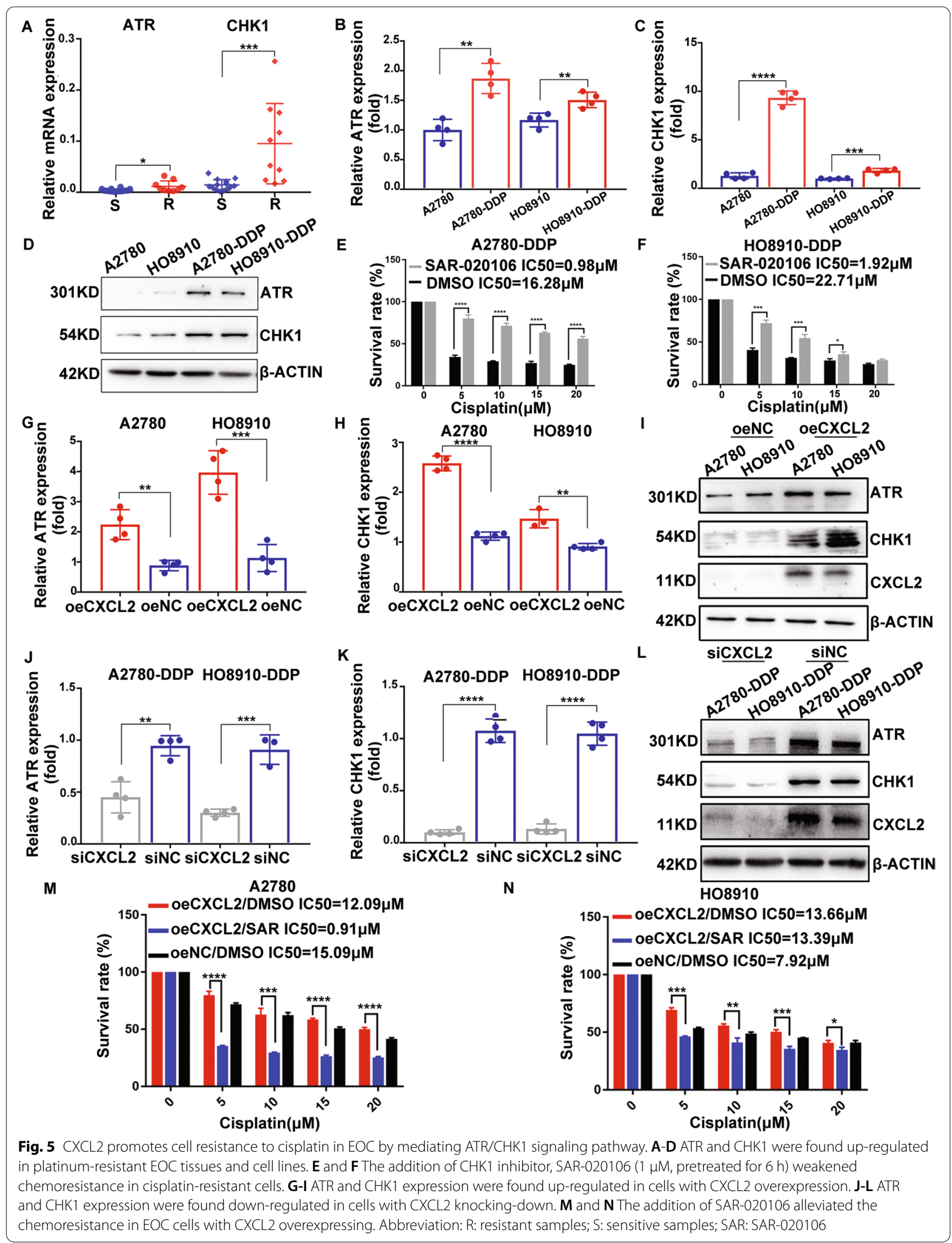


novel regulation mechanism of CXCL2 in EOC platinum resistance by mediating ATR/CHK1 signaling pathway. However, by analyzing the gene data of GSE114206 dataset and TCGA database, it showed no statistically significant in correlation of CXCL2 and ATR/CHK1 expression in ovarian tumor. These results might be caused by the complex gene regulation network in cancer, and the interactions between genes may not be singular.

\section{Conclusions}

In summary, this study revealed the crucial role of CXCL2 and demonstrated a new regulatory mechanism of CXCL2-mediated ATR/CHK1 signaling pathway in platinum-resistant EOC. Our findings suggest that blocking the role of CXCL2 and its regulatory signaling pathways represents a potential approach to overcome platinum resistance in EOC.

\section{Abbreviations \\ EOC: Epithelial ovarian cancer; DE: Differentially expressed; DEGs: Differentially expressed genes; CCK8: Cell Counting Kit-8; qRT-PCR: RNA extraction and quantitative Real-time PCR; ELISA: Enzyme-linked immunosorbent assay; TCGA :The Cancer Genome Atlas; TME: Tumor microenvironment; DDR: DNA dam- age repairment.}

\section{Supplementary Information}

The online version contains supplementary material available at https://doi. org/10.1186/s13048-021-00864-3.

Additional file 1: Supplementary Figure 1. The correlation of CXCL2 and ATR/CHK1 gene expression in EOC. (A) The correlation analyses of CXCL2 and ATR/CHK1 based on gene expression profile of GSE114206 showed no statistical significance. (B and C)The correlation analyses of CXCL2 and ATR/CHK1 based on TCGA database also showed no statistical significance. Abbreviation: TCGA: The Cancer Genome Atlas.

Additional file 2: Supplementary Table 1: The sequences of gene primers used for QRT-PCR

Additional file 3: Supplementary Table 2.

\section{Acknowledgements}

We thank all members of the Cheng's laboratory for their advice and technical assistance.

\section{Authors' contributions}

Conceptualization, Wenjun Cheng, Sipei Nie; Methodology, Sipei Nie and Yicong Wan; Bioinformatics Analysis, Jinhui Liu; Writing -Original Draft, Sipe Nie and Hui Wang; Writing -Review \& Editing, Jing Yang and Rui sun; Funding Acquisition, Wenjun Cheng; Supervision, Huangyang Meng, Xiaolin Ma and Yi Jiang. All authors read and approved the final manuscript.

\section{Funding}

This work was supported by the National Natural Science Foundation of China (81872119).

\section{Availability of data and materials}

They could be achieved upon reasonable request to the authors.

\section{Declarations}

Ethics approval and consent to participate

All patients signed informed consent before using clinical specimens. The use of specimens for this study has been proved by the ethics committee of the First Affiliated Hospital with Nanjing Medical University.

\section{Consent for publication}

Not applicable.

\section{Competing interests}

The authors declare that they have no competing interests.

Received: 30 March 2021 Accepted: 18 August 2021

Published online: 03 September 2021

\section{References}

1. Siegel RL, Miller KD, Jemal A. Cancer statistics, 2020. CA Cancer J Clin. 2020;70:7-30.

2. Lheureux S, Braunstein M, Oza AM. Epithelial ovarian cancer: Evolution of management in the era of precision medicine. CA Cancer J Clin. 2019:69:280-304.

3. van Zyl B, Tang D, Bowden NA. Biomarkers of platinum resistance in ovarian cancer: what can we use to improve treatment. Endocr Relat Cancer. 2018;25:R303-18

4. Caligiuri A, Pastore M, Lori G, Raggi C, Di Maira G, Marra F, et al. Role of Chemokines in the Biology of Cholangiocarcinoma. Cancers (Basel). 2020;12(8).

5. Morein D, Erlichman N, Ben-Baruch A. Beyond Cell Motility: The Expanding Roles of Chemokines and Their Receptors in Malignancy. Front Immunol. 2020;11:952.

6. Nagarsheth N, Wicha MS, Zou W. Chemokines in the cancer microenvironment and their relevance in cancer immunotherapy. Nat Rev Immunol. 2017;17:559-72.

7. Peng D, Kryczek I, Nagarsheth N, Zhao L, Wei S, Wang W, et al. Epigenetic silencing of TH1-type chemokines shapes tumour immunity and immunotherapy. Nature. 2015;527:249-53.

8. Galluzzi L, Humeau J, Buque A, Zitvogel L, Kroemer G. Immunostimulation with chemotherapy in the era of immune checkpoint inhibitors. Nat Rev Clin Oncol. 2020;17(12):725-41.

9. Perez-Ruiz E, Melero I, Kopecka J, Sarmento-Ribeiro AB, Garcia-Aranda M, De Las RJ. Cancer immunotherapy resistance based on immune checkpoints inhibitors: Targets, biomarkers, and remedies. Drug Resist Updat. 2020:53:100718.

10. Scala S. Molecular Pathways: Targeting the CXCR4-CXCL12 AxisUntapped Potential in the Tumor Microenvironment. Clin Cancer Res. 2015;21:4278-85.

11. Ishida T, Ishii T, Inagaki A, Yano H, Kusumoto S, Ri M, et al. The CCR4 as a novel-specific molecular target for immunotherapy in Hodgkin lymphoma. Leukemia. 2006:20:2162-8.

12. Ito A, Ishida T, Yano H, Inagaki A, Suzuki S, Sato F, et al. Defucosylated anti-CCR4 monoclonal antibody exercises potent ADCC-mediated antitumor effect in the novel tumor-bearing humanized NOD/Shiscid, IL-2Rgamma(null) mouse model. Cancer Immunol Immunother. 2009:58:1195-206.

13. Kitadate A, Ikeda S, Abe F, Takahashi N, Shimizu N, Matsue K, et al. Histone deacetylase inhibitors downregulate CCR4 expression and decrease mogamulizumab efficacy in CCR4-positive mature T-cell lymphomas. Haematologica. 2018;103:126-35.

14. Ahmed H, Ghoshal A, Jones S, Ellis I, Islam M. Head and Neck Cancer Metastasis and the Effect of the Local Soluble Factors, from the Microenvironment, on Signalling Pathways: Is It All about the Akt? Cancers (Basel). 2020;12(8).

15. Thomas RP, Nagpal S, Iv M, Soltys SG, Bertrand S, Pelpola JS, et al. Macrophage Exclusion after Radiation Therapy (MERT): A First in Human Phase I/II Trial using a CXCR4 Inhibitor in Glioblastoma. Clin Cancer Res. 2019;25:6948-57 
16. Layer JP, Kronmuller MT, Quast T, van den Boorn-Konijnenberg D, Effern M, Hinze D, et al. Amplification of N-Myc is associated with a T-cell-poor microenvironment in metastatic neuroblastoma restraining interferon pathway activity and chemokine expression. Onco Immunol. 2017;6:e1320626.

17. Choreno-Parra JA, Thirunavukkarasu S, Zuniga J, Khader SA. The protective and pathogenic roles of CXCL17 in human health and disease: Potential in respiratory medicine. Cytokine Growth Factor Rev. 2020;53:53-62.

18. Ren Y, Gu YK, Li Z, Xu GZ, Zhang YM, Dong MX, et al. CXCR3 confers sorafenib resistance of HCC cells through regulating metabolic alteration and AMPK pathway. Am J Transl Res. 2020;12:825-36.

19. Zhang G, Luo X, Zhang W, Chen E, Xu J, Wang F, et al. CXCL-13 Regulates Resistance to 5-Fluorouracil in Colorectal Cancer. Cancer Res Treat. 2020;52:622-33.

20. Wang J, Dean DC, Hornicek FJ, Shi H, Duan Z. RNA sequencing (RNA-Seq) and its application in ovarian cancer. Gynecol Oncol. 2019;152:194-201.

21. Armstrong DK, Alvarez RD, Bakkum-Gamez JN, Barroilhet L, Behbakht K, Berchuck A, et al. NCCN Guidelines Insights: Ovarian Cancer, Version 1.2019. J Natl Compr Canc Netw. 2019;17:896-909.

22. Ding J, Xu K, Zhang J, Lin B, Wang Y, Yin S, et al. Overexpression of CXCL2 inhibits cell proliferation and promotes apoptosis in hepatocellular carcinoma. BMB Rep. 2018:51:630-5.

23. Jiang Y, Wan Y, Gong M, Zhou S, Qiu J, Cheng W. RNA demethylase ALKBH5 promotes ovarian carcinogenesis in a simulated tumour microenvironment through stimulating NF-kappaB pathway. J Cell Mol Med. 2020;24:6137-48.

24. Velaei K, Samadi N, Barazvan B, Soleimani RJ. Tumor microenvironmentmediated chemoresistance in breast cancer. Breast. 2016;30:92-100.

25. Bikfalvi A, Billottet C. The CC and CXC chemokines: major regulators of tumor progression and the tumor microenvironment. Am J Physiol Cell Physiol. 2020;318:C542-54.

26. Vandercappellen J, Van Damme J, Struyf S. The role of CXC chemokines and their receptors in cancer. Cancer Lett. 2008;267:226-44.

27. Yu PF, Huang Y, Han YY, Lin LY, Sun WH, Rabson AB, et al. TNFalpha-activated mesenchymal stromal cells promote breast cancer metastasis by recruiting CXCR2(+) neutrophils. Oncogene. 2017;36:482-90.

28. Li X, Chen Y, Chang Y, Li S, Zhao Z, Zhang H. CXCR2 is involved in pulmonary intravascular macrophage accumulation and angiogenesis in a rat model of hepatopulmonary syndrome. Clin Sci (Lond). 2017;131:159-68.

29. Acharyya S, Oskarsson T, Vanharanta S, Malladi S, Kim J, Morris PG, et al. A CXCL1 paracrine network links cancer chemoresistance and metastasis. Cell. 2012;150:165-78.

30. Natsume M, Shimura T, Iwasaki H, Okuda Y, Hayashi K, Takahashi S, et al. Omental adipocytes promote peritoneal metastasis of gastric cancer through the CXCL2-VEGFA axis. Br J Cancer. 2020;123:459-70.
31. Peng ZP, Jiang ZZ, Guo HF, Zhou MM, Huang YF, Ning WR, et al. Glycolytic activation of monocytes regulates the accumulation and function of neutrophils in human hepatocellular carcinoma. J Hepatol. 2020;73(4):906-17.

32. Ye Q, Zhai X, Wang W, Zhang S, Zhu H, Wang D, et al. Overexpression of Growth-Related Oncogene-beta Is Associated with Tumorigenesis, Metastasis, and Poor Prognosis in Ovarian Cancer. Dis Markers. 2015;2015:387382.

33. Taki M, Abiko K, Baba T, Hamanishi J, Yamaguchi K, Murakami R, et al. Snail promotes ovarian cancer progression by recruiting myeloid-derived suppressor cells via CXCR2 ligand upregulation. Nat Commun. 2018;9:1685.

34. Galluzzi L, Senovilla L, Vitale I, Michels J, Martins I, Kepp O, et al. Molecular mechanisms of cisplatin resistance. Oncogene. 2012;31:1869-83.

35. Sancar A, Lindsey-Boltz LA, Unsal-Kacmaz K, Linn S. Molecular mechanisms of mammalian DNA repair and the DNA damage checkpoints. Annu Rev Biochem. 2004;73:39-85.

36. Myers K, Gagou ME, Zuazua-Villar P, Rodriguez R, Meuth M. ATR and Chk1 suppress a caspase-3-dependent apoptotic response following DNA replication stress. PLOS Genet. 2009;5:e1000324.

37. Gatei M, Kijas AW, Biard D, DorkT, Lavin MF. RAD50 phosphorylation promotes ATR downstream signaling and DNA restart following replication stress. Hum Mol Genet. 2014;23:4232-48.

38. Sun B, Ross SM, Rowley S, Adeleye Y, Clewell RA. Contribution of ATM and ATR kinase pathways to p53-mediated response in etoposide and methyl methanesulfonate induced DNA damage. Environ Mol Mutagen. 2017;58:72-83.

39. Rundle S, Bradbury A, Drew Y, Curtin NJ. Targeting the ATR-CHK1 Axis in Cancer Therapy. Cancers (Basel). 2017;9(5).

40. Reinhardt HC, Yaffe MB. Kinases that control the cell cycle in response to DNA damage: Chk1, Chk2, and MK2. Curr Opin Cell Biol. 2009;21:245-55.

41. Qiu Z, Oleinick NL, Zhang J. ATR/CHK1 inhibitors and cancer therapy. Radiother Oncol. 2018;126:450-64.

42. Huntoon CJ, Flatten KS, Wahner HA, Huehls AM, Sutor SL, Kaufmann SH, et al. ATR inhibition broadly sensitizes ovarian cancer cells to chemotherapy independent of BRCA status. Cancer Res. 2013;73:3683-91.

\section{Publisher's Note}

Springer Nature remains neutral with regard to jurisdictional claims in published maps and institutional affiliations.
Ready to submit your research? Choose BMC and benefit from:

- fast, convenient online submission

- thorough peer review by experienced researchers in your field

- rapid publication on acceptance

- support for research data, including large and complex data types

- gold Open Access which fosters wider collaboration and increased citations

- maximum visibility for your research: over 100M website views per year

At BMC, research is always in progress.

Learn more biomedcentral.com/submissions 